MATHEMATICS OF COMPUTATION

Volume 75, Number 254, Pages 673-696

S $0025-5718(06) 01788-1$

Article electronically published on January 23, 2006

\title{
CONVOLUTION QUADRATURE TIME DISCRETIZATION OF FRACTIONAL DIFFUSION-WAVE EQUATIONS
}

\author{
EDUARDO CUESTA, CHRISTIAN LUBICH, AND CESAR PALENCIA
}

\begin{abstract}
We propose and study a numerical method for time discretization of linear and semilinear integro-partial differential equations that are intermediate between diffusion and wave equations, or are subdiffusive. The method uses convolution quadrature based on the second-order backward differentiation formula. Second-order error bounds of the time discretization and regularity estimates for the solution are shown in a unified way under weak assumptions on the data in a Banach space framework. Numerical experiments illustrate the theoretical results.
\end{abstract}

\section{INTRODUCTION}

In this paper we study time discretization of a class of integro-partial differential equations whose prototype equation is, for $0<\beta<1$,

$$
\frac{\partial u}{\partial t}(x, t)=\frac{1}{\Gamma(\beta)} \int_{0}^{t}(t-\tau)^{\beta-1} \Delta u(x, \tau) d \tau+F(x, t, u(x, t), \nabla u(x, t))
$$

for $x$ in a domain $\Omega \subset \mathbf{R}^{d}$ and $t>0$, taken together with Dirichlet or Neumann boundary conditions and with the initial condition $u(x, 0)=u_{0}(x)$ for $x \in \Omega$.

Since the integral term can be viewed as the $\beta$-th integral [32, 34] of $\Delta u(x, \cdot)$, equation (1.1) is intermediate between the diffusion $(\beta=0)$ and the wave $(\beta=1)$ equation [9, 10], and it can be termed a fractional PDE of order $\alpha=\beta+1 \epsilon$ $(1,2)$ in time. Equations such as (1.1) describe anomalous diffusion processes and wave propagation in viscoelastic materials, e.g., [12, 14, 25, 28, 29, 33. They have recently attracted increasing interest in the physical, chemical and engineering literature (see the numerous papers citing [28] or [32]).

Numerical methods for the time discretization of (1.1) have been proposed by various authors $4,5,17,8,17,24,26,35,36,37$. A usual approach for the time discretization of (1.1) consists in treating separately the derivative and the integral term, which are approximated by a standard difference formula and by means of a suitable quadrature rule, respectively. The traditional analysis of the error, based on consistency and stability, shows that this approach is likely to exhibit a severe order reduction, the reason being the lack of regularity in time of the solution, which is present even in the case of smooth initial data. Precisely, one of the main

Received by the editor January 20, 2004 and, in revised form, September 29, 2004.

2000 Mathematics Subject Classification. Primary 65R20, 65M15; Secondary 26A33, 45K05.

Key words and phrases. Anomalous diffusion, parabolic equation with memory, time discretization, convolution quadrature, fractional BDF method, error analysis, regularity.

The first and third authors were supported by Grant MCYT BFM2001-2013 cofinanced by FEDER funds. The second author was supported by DFG SFB 382. 
goals of the present paper is to derive a systematic and computationally affordable approach to render the time integration of (at least) second order of accuracy under realistic, weak regularity assumptions.

The paper considers the fractional version of the second-order backward differentiation formula (BDF2) [18, 21], although there are no real difficulties in extending the scope so as to cover higher-order BDFs. To overcome the order reduction it is necessary to carry out some corrections to the basic method. One way of doing this is by corrections that eliminate the consistency error of the nonsmooth terms in the expansion of $u$ at $t=0$ [18, 19]. However, in the framework of the traditional error analysis based on the consistency of the approximation to the integral term and on stability, this approach still requires strong spatial regularity and high compatibility with the boundary conditions. Moreover, the smoothing properties of the equation are not taken into account.

The main tool used in this paper is convolution quadrature 21, 22], which enables us to treat the time discretization as a whole, i.e., combining the approximations to the derivative and to the integral in (1.1). Convolution quadrature allows for a direct estimate of the error in the linear problem, based on resolvent bounds of the elliptic operator. Stability is then derived as a corollary. The error analysis uses the regularity of the data rather than that of the solution. Finally, convolution quadrature permits us to introduce in a clear and direct way a specific treatment of the initial condition and the inhomogeneity which results in an optimal error estimate, also when the solution fails to possess the regularity required in the traditional approach. (Cf. 24] for preliminary results along such lines.)

In the paper, equation (1.1) is written as an abstract, parabolic, evolution equation in a Banach space. This allows us to consider general elliptic operators other than the Laplacian and to study the errors in $L^{p}$-norms, $1 \leq p \leq+\infty$. The precise analytical framework and notation are given in Section 2, while convolution quadrature is presented, in a self-contained form, in Section 3. The numerical method is considered first in Section 4, in the setting of linear, non-homogeneous problems. Here, together with basic estimates for smooth and non-smooth data, auxiliary estimates in fractional norms, needed for non-linear problems, are also established. In Section 5 we comment on the class of non-linear problems we consider, as well as on the regularity of their solutions. In Section 6 we introduce and analyze the numerical method for non-linear problems. This section makes an extensive use of the results for the linear case given in Section 4. Since the lack of regularity of the solutions is one of the main motivations of the paper, we have included the proof of the regularity results in Section 7. The regularity analysis and the error analysis of the numerical method are both based on the operational calculus in a unified way. In Section 8 we consider the adaptation of the method to the situation of subdiffusion, which replaces the integral term in (1.1) by its time derivative, and in this way corresponds to $\alpha=\beta+1 \in(0,1)$. The final Section 9 gives some numerical illustrations.

\section{ANALYTICAL FRAMEWORK AND NOTATION}

2.1. Abstract setting. We consider (1.1) as an evolution equation on a Banach space $X$ (such as $X=L_{p}(\Omega)$ ) and study the abstract initial-value problem on $X$,

$$
u^{\prime}(t)+\frac{1}{\Gamma(\beta)} \int_{0}^{t}(t-\tau)^{\beta-1} A u(\tau) d \tau=f(t), \quad 0<t \leq T, \quad u(0)=u_{0}
$$


where $0<\beta<1, u_{0} \in X$, and $t \mapsto f(t) \in X$ is a continuous inhomogeneity. Later we will turn to the semilinear problem where $f(t)$ is replaced by a nonlinearity $F(t, u(t))$. We assume that $-A$ is a sectorial operator on $X$ [13, 31]; that is, a densely defined closed operator on $X$ whose resolvent is analytic in a sector $|\arg (\lambda-a)|<\theta$ with $\theta>\frac{1}{2} \pi$ and bounded by

$$
\left\|(\lambda+A)^{-1}\right\| \leq \frac{M_{0}}{|\lambda|} \quad \text { for } \quad|\arg (\lambda-a)|<\theta .
$$

We will assume $a<0$ for convenience (because we can then use fractional powers of $A$ instead of those of $A+\sigma I$ for some $\sigma>a$ ) and, as an essential condition,

$$
\theta>\frac{1}{2} \pi \alpha \quad \text { with } \quad \alpha=\beta+1 .
$$

Let us mention examples of operators $A$ that fall into this framework and that are used in the physics literature; see for example [13, 28, 31]:

- the negative Laplacian $A=-\Delta$ on $\mathbf{R}^{d}$ or on a domain $\Omega \subset \mathbf{R}^{d}$ together with Dirichlet or Neumann boundary conditions,

- fractional powers of the negative Laplacian on $\mathbf{R}^{d}, A=(-\Delta)^{\nu / 2}$ with $\nu>0$,

- the Fokker-Planck operator given by $A u=-\Delta u-\nabla \cdot(u \nabla V)$ for a smooth potential $V$.

Typically, the space discretizations of such operators also satisfy the resolvent bound (2.2), with a constant $M_{0}$ and angle $\theta$ independent of the space discretization parameter; see, e.g., [1, 2].

The integral operator in (2.1) is the fractional integral of order $\beta$, and with $\partial$ symbolizing time differentiation, the problem (2.1) is written more compactly as

$$
u^{\prime}+\partial^{-\beta} A u=f, \quad u(0)=u_{0},
$$

or upon integrating from 0 to $t$ and writing $\alpha=\beta+1$, as

$$
u+\partial^{-\alpha} A u=u_{0}+\partial^{-1} f .
$$

In most of the paper we consider this equation in the situation

$$
1<\alpha<2 \text {. }
$$

The case $0<\alpha<1$ (which corresponds to taking the derivative of the integral term in (2.1)) is equally of interest, and the extension of our results to this case is given in Section 8. Equation (2.4) will be the starting point for further development. Before that, it is convenient to extend the operational notation.

2.2. Operational calculus notation. Let $K(s)$ be a complex-valued or operatorvalued function that is analytic in a sector with opening angle to the positive real axis greater than $\frac{1}{2} \pi$, and there bounded by

$$
\|K(s)\| \leq M|s-\sigma|^{-\mu} \quad \text { for } \quad|\arg (s-\sigma)|<\varphi \text {, with } \varphi>\frac{1}{2} \pi,
$$

for some real $\mu, M$, and $\sigma$. Then, $K(s)$ is the Laplace transform of a distribution $k$ on the real line which vanishes for $t<0$, has its singular support empty or concentrated in $t=0$, and which is an analytic function for $t>0$. If $\mu \leq 0$ is an integer, then the singular part of $k$ is determined by the polynomial part $P(s)$ of $K(s)$ for $s \rightarrow \infty$ and given as $P(\partial) \delta_{0}$, where $\partial$ again denotes time differentiation 
and $\delta_{0}$ is Dirac's delta at $t=0$. For $t>0$, the analytic function $k(t)$ is given by the inversion formula

$$
k(t)=\frac{1}{2 \pi i} \int_{\Gamma} K(s) e^{s t} d s, \quad t>0,
$$

with $\Gamma$ a contour in the sector of analyticity, parallel to its boundary and oriented with increasing imaginary part. On substituting $w=(s-\sigma) t$ in the integral and replacing the resulting contour for $w \in t(\Gamma-\sigma)$ by an equivalent, $t$-independent contour, this formula and the bound (2.6) immediately imply the following bound, which we formulate as a lemma because it is used frequently.

Lemma 2.1. If $K(s)$ is analytic and bounded by (2.6), then there exists $B>0$, which depends only on $M, \mu$, and $\varphi$, such that the inverse Laplace transform $k(t)$ is bounded by

$$
\|k(t)\| \leq B t^{\mu-1} e^{\sigma t} \quad \text { for } \quad t>0
$$

In particular, $k(t)$ is locally integrable if $\mu>0$.

We define $K(\partial)$ as the operator of (distributional) convolution with the kernel $k$ : $K(\partial) g=k * g$. If $\mu>0$, this is given by the convolution integral

$$
K(\partial) g(t)=\int_{0}^{t} k(\tau) g(t-\tau) d \tau, \quad t>0
$$

for functions $g(t)$ that are continuous in $t \geq 0$ (and extended by 0 to $t<0$ ) and for $\mu>-m$ ( $m$ a positive integer) by the differentiated convolution integral, with $\widetilde{K}(s)=s^{-m} K(s)$,

$$
K(\partial) g(t)=\partial^{m} \widetilde{K}(\partial) g(t)=\left(\frac{d}{d t}\right)^{m} \int_{0}^{t} \widetilde{k}(\tau) g(t-\tau) d \tau, \quad t>0,
$$

for functions $g(t)$ that are $m$-times continuously differentiable in $t \geq 0$. This notation extends the fractional-order differentiation notation already used above. For two functions $K_{1}(s)$ and $K_{2}(s)$ of the above type, the associativity of convolution and the convolution rule of Laplace transforms give us the basic relation

$$
K_{2}(\partial) K_{1}(\partial) g=\left(K_{2} K_{1}\right)(\partial) g \text {. }
$$

An equivalent identity is

$$
K_{2}(\partial)\left(k_{1} * g\right)=\left(K_{2}(\partial) k_{1}\right) * g .
$$

2.3. Resolvent bounds. In the present paper, the role of $K(s)$ will be taken by $s^{-1}\left(I+s^{-\alpha} A\right)^{-1}$ and by $A^{\nu}\left(I+s^{-\alpha} A\right)^{-1}$ for various fractional powers $\nu$. Here we have the following bounds.

Lemma 2.2. For a sectorial operator with (2.2), (2.3) there is a constant $M$, which depends only on $M_{0}$ and $\theta$ in (2.2), such that the following bounds hold in the operator norm on $X$, uniformly for $|\arg (s)|<\theta / \alpha$ (by (2.3), $\left.\theta / \alpha>\frac{1}{2} \pi\right)$ :

$$
\begin{aligned}
\left\|A^{\nu}\left(I+s^{-\alpha} A\right)^{-1}\right\| & \leq M|s|^{\alpha \nu}, & & \leq \nu \leq 1, \\
\left\|A^{\nu}\left(I+s^{-\alpha} A\right)^{-1}-A^{\nu}\right\| & \leq M|s|^{\alpha \nu}, & & -1 \leq \nu \leq 0, \\
\left\|A^{\nu}\left(I+s^{-\alpha} A\right)^{-1}-A^{\nu}+s^{-\alpha} A^{\nu+1}\right\| & \leq M|s|^{\alpha \nu}, & & -2 \leq \nu \leq-1 .
\end{aligned}
$$


Proof. For $0 \leq \nu \leq 1$ we write

$$
A^{\nu}\left(I+s^{-\alpha} A\right)^{-1}=s^{\alpha \nu} \cdot\left(s^{-\alpha} A\right)^{\nu}\left(I+s^{-\alpha} A\right)^{-1},
$$

which yields the estimate; see [13, Chap. 1]. For $-1 \leq \nu \leq 0$ we use the identity

$$
\left(I+s^{-\alpha} A\right)^{-1}=I-s^{-\alpha} A\left(I+s^{-\alpha} A\right)^{-1}
$$

so that

$$
A^{\nu}\left(I+s^{-\alpha} A\right)^{-1}=A^{\nu}-s^{-\alpha} A^{\nu+1}\left(I+s^{-\alpha} A\right)^{-1},
$$

and the stated bound thus follows from the previous case. For $-2 \leq \nu \leq-1$ we use the identity (2.16) twice to obtain

$$
A^{\nu}\left(I+s^{-\alpha} A\right)^{-1}=A^{\nu}-s^{-\alpha} A^{\nu+1}+s^{-2 \alpha} A^{\nu+2}\left(I+s^{-\alpha} A\right)^{-1},
$$

which reduces the result again to the first case.

2.4. Mild solutions and regularity. When we apply $K(\partial)=\left(I+\partial^{-\alpha} A\right)^{-1}$ to both sides of (2.4), it follows from (2.11) that every solution $u \in C^{1}([0, T], X) \cap$ $C([0, T], D(A))$ of (2.4) is given by

$$
u=\left(I+\partial^{-\alpha} A\right)^{-1}\left(u_{0}+\partial^{-1} f\right) .
$$

This formula expresses $u$ as a temporal convolution and also makes sense under weak regularity assumptions on the data, e.g., $u_{0} \in X$ and $f \in L^{1}(0, T ; X)$, which do not imply the above-mentioned regularity of $u$. The formula (2.17) then defines a generalized or mild solution of (2.4) or (2.1). We mention in passing that (2.17) can be rewritten as

$$
u(t)=k(t) u_{0}+\int_{0}^{t} k(t-\tau) f(\tau) d \tau
$$

where $k(t)$ is the inverse Laplace transform of $K(s)=s^{-1}\left(I+s^{-\alpha} A\right)^{-1}$. The bounds of Lemma 2.2, used together with Lemma 2.1, give regularity estimates of the mild solution. For example, we obtain the following result with regularity conditions on the data that we will consider repeatedly in this paper. Here and in what follows, $X_{\rho}$ denotes, for $\rho \geq 0$, the domain of $A^{\rho}$ :

$$
X_{\rho}=D\left(A^{\rho}\right) \quad \text { with norm }\|v\|_{\rho}=\left\|A^{\rho} v\right\| .
$$

Lemma 2.3. If $u_{0} \in X_{2 / \alpha}, f(0) \in X_{1 / \alpha}$, and $f \in C^{2}([0, T], X)$, then the mild solution $u$ defined by (2.17) is of the form

$$
u(t)=u_{0}+t f(0)-\frac{t^{\alpha}}{\Gamma(\alpha+1)} A u_{0}+v(t),
$$

where $v \in C^{2}([0, T], X)$ with $v(0)=v^{\prime}(0)=0$. Moreover, for $\rho$ with $0 \leq \alpha \rho<1$, we have $u \in C^{2}\left((0, T], X_{\rho}\right)$ with $u(0)=u_{0}, u^{\prime}(0)=f(0)$, and

$$
\left\|u^{\prime \prime}(t)\right\|_{\rho} \leq C t^{-r} \quad \text { with } \quad r=\max (\alpha \rho, 2-\alpha)<1 .
$$

The proof consists of several applications of Lemmas 2.2 and 2.1 and is given in Section 7 . 


\section{Convolution quadrature}

A convolution quadrature [21, 22] approximates the continuous convolution $K(\partial) g(t)$ by a discrete convolution with a time step size $h>0$,

$$
K\left(\partial_{h}\right) g(t)=\sum_{0 \leq j h \leq t} \omega_{j} g(t-j h), \quad t>0,
$$

where the quadrature weights $\omega_{j}$ are determined by their generating power series

$$
\sum_{j=0}^{\infty} \omega_{j} \zeta^{j}=K\left(\frac{\delta(\zeta)}{h}\right)
$$

Here $K(s)$ is again the Laplace transform of the convolution kernel, and $\delta(\zeta)$ is a rational function, chosen as the quotient of the generating polynomials of a linear multistep method. For concreteness, in this paper we consider the second-order backward differentiation formula, for which

$$
\delta(\zeta)=(1-\zeta)+\frac{1}{2}(1-\zeta)^{2}
$$

The discrete kernel given by

$$
\kappa_{n}=\omega_{n} / h
$$

satisfies a bound of the same type as the continuous kernel.

Lemma 3.1 ([22, (2.6)] (or [21, (5.4)] in the case $\mu>0)$ ). Under condition (2.6) we have the analogue of (2.8): there exist $B>0$ and $\widetilde{\sigma}$ of the same sign as $\sigma$, which depend only on $M, \mu, \varphi$, and $\sigma$, such that

$$
\left\|\kappa_{n}\right\| \leq B t^{\mu-1} e^{\widetilde{\sigma} t} \quad \text { for } \quad t=n h, \quad n=1,2,3, \ldots .
$$

For $n=0$, (3.2) and (2.6) directly imply $\left\|\kappa_{0}\right\|=\|K(\delta(0) / h) / h\| \leq B h^{\mu-1}$.

An important property is that the relations (2.11) and (2.12) are maintained in the discretization:

$$
\begin{gathered}
K_{2}\left(\partial_{h}\right) K_{1}\left(\partial_{h}\right) g=\left(K_{2} K_{1}\right)\left(\partial_{h}\right) g, \\
K_{2}\left(\partial_{h}\right)\left(k_{1} * g\right)=\left(K_{2}\left(\partial_{h}\right) k_{1}\right) * g .
\end{gathered}
$$

Note, however, that (3.5) and (3.6) are not the same expressions, in contrast to their continuous analogues. While (3.6) holds for any choice of weights in (3.1), the relation (3.5) requires a construction of the type (3.2).

The approximation properties of (3.1) -3.3 are described in the following result, which considers the particular, but essential case $g(t)=t^{\gamma-1} c$ for real $\gamma>0$, with a time-independent $c$, a scalar or vector or operator as needed. Here we often write $K\left(\partial_{h}\right) g(t)=K\left(\partial_{h}\right) \tau^{\gamma-1} c(t)$, where $\tau(t)=t$ is the symbol of the identity function. (For $\gamma<1$ the sum in (3.1) is understood to be over $0 \leq j h \leq t-h$ to avoid the evaluation of $g$ very close to the singularity at 0 .)

Lemma 3.2 ([22, Theorem 2.2] (or [21, Theorem 5.2] in the case $\mu>0)$ ). Assume that $K(s)$ is analytic and bounded as in (2.6), and let $g(t)=t^{\gamma-1} c$. Then, the error of the convolution quadrature approximation (3.1) with (3.2), based on the second-order backward differentiation formula (3.3), is bounded by

$$
\left\|K\left(\partial_{h}\right) g(t)-K(\partial) g(t)\right\| \leq\left\{\begin{array}{lr}
C t^{\mu-1} h^{\gamma}, & 0<\gamma \leq 2 \\
C t^{\mu+\gamma-3} h^{2}, & \gamma \geq 2
\end{array}\right.
$$

where the constant $C$ does not depend on $h$ and $t \in(0, T]$ with fixed $T<\infty$. 
In view of (3.6) and (2.12), this result yields an estimate of the approximation error for all functions $g(t)$ which can be written as an expansion in powers of $t$ with a remainder term that can be expressed as a convolution of a power function with a locally integrable function. In particular, this is the case for the Taylor expansion of smooth functions with the remainder term in integral form:

$$
g(t)=g(0)+t g^{\prime}(0)+\tau * g^{\prime \prime}(t) \quad(\text { with } \tau(t)=t) .
$$

Note that $\left(K\left(\partial_{h}\right)-K(\partial)\right)\left(\tau * g^{\prime \prime}\right)(t)=\left(\left(K\left(\partial_{h}\right)-K(\partial)\right) \tau I\right) * g^{\prime \prime}(t)$.

Here, the problem is that the constant term $c=g(0)$ yields only a first-order error bound $(\gamma=1)$ :

$$
\left\|K\left(\partial_{h}\right) 1 c(t)-K(\partial) 1 c(t)\right\| \leq C t^{\mu-1} h .
$$

This causes difficulty unless $K(\partial) 1 c(t)=\int_{0}^{t} k(\tau) c d \tau$ is available analytically. We describe two general approaches that improve the situation. In the first approach, we write for some $\nu \geq 1$ the identity

$$
K(\partial) 1 c(t)=K(\partial) \partial^{\nu}\left(\partial^{-\nu} 1\right) c(t)=K(\partial) \partial^{\nu} \frac{\tau^{\nu}}{\Gamma(\nu+1)} c(t)
$$

By Lemma 3.2 with $\gamma=\nu+1 \geq 2$ and $\mu$ replaced by $\mu-\nu$, we thus obtain a second-order approximation to $K(\partial) 1 c(t)$ :

$$
\left\|K\left(\partial_{h}\right) \partial_{h}^{\nu} \frac{\tau^{\nu}}{\Gamma(\nu+1)} c(t)-K(\partial) 1 c(t)\right\| \leq C t^{\mu-2} h^{2} .
$$

The second approach, which considers approximations only at gridpoints $t_{n}=n h$, takes half the value of the function $g$ at $t=0$, or in other terms, replaces $g$ by $1_{h} g$, where $1_{h}(t)$ is a function with $1_{h}(0)=\frac{1}{2}$ and $1_{h}(t)=1$ for $t \geq h$. An alternative, sometimes more convenient, choice is $1_{h}(0)=0,1_{h}(h)=\frac{3}{2}$, and $1_{h}(t)=1$ for $t \geq 2 h$. We then take the initial-point correction

$$
K\left(\partial_{h}\right)\left(1_{h} g\right)\left(t_{n}\right)=\omega_{n} 1_{h}(0) g(0)+\omega_{n-1} 1_{h}(h) g(h)+\cdots+\omega_{0} g\left(t_{n}\right)
$$

as the approximation instead of $K\left(\partial_{h}\right) g\left(t_{n}\right)$.

The second choice of $1_{h}$ turns out to yield $1_{h}=\partial_{h} \partial^{-1} 1$ at gridpoints $t_{n}$, so that here the second approach coincides with the first approach with $\nu=1$.

More generally, a possible choice is $1_{h}\left(t_{n}\right)=q_{n}$ where $q_{n}=1+O\left(r^{n}\right)$, with $r<1$ and $h \sum_{0}^{\infty} q_{n} e^{-n h}=1+O\left(h^{2}\right)$. In particular, this holds for $\sum_{0}^{\infty} q_{n} \zeta^{n}=\delta(\zeta)^{-1}$. For every such choice of $1_{h}$, [22, Theorem 2.1] (or [21, Theorem 4.1] in the case $\mu>0$ ) gives us, with the proof of [21, Corollary 4.2], a bound of the same type as (3.8):

$$
\left\|K\left(\partial_{h}\right) 1_{h} c(t)-K(\partial) 1 c(t)\right\| \leq C t^{\mu-2} h^{2} \quad \text { at } t=t_{n}
$$

\section{Time Discretization of THE LineAR EQUATION}

4.1. Construction of the numerical method. We consider a numerical method for the time discretization of (2.1), constructed with the convolution quadrature based on the second-order backward difference formula. In view of the causal nature of both the method and the error estimates we will obtain, there is no loss of generality in assuming that $f:[0,+\infty) \rightarrow X$. Starting with the integral equation (2.4), viz.,

$$
u+\partial^{-\alpha} A u=u_{0}+\partial^{-1} f
$$


suggests that we consider the numerical approximation $u^{h}:[0,+\infty) \rightarrow X, h>0$, to $u$ defined by the discrete equation

$$
u^{h}+\partial_{h}^{-\alpha} A u^{h}=u_{0}+\partial_{h}^{-1} f
$$

By (3.5) this is equivalent to the direct convolution quadrature discretization of the solution formula (2.17), viz.,

$$
u=\left(I+\partial^{-\alpha} A\right)^{-1}\left(u_{0}+\partial^{-1} f\right),
$$

by

$$
u^{h}=\left(I+\partial_{h}^{-\alpha} A\right)^{-1}\left(u_{0}+\partial_{h}^{-1} f\right) .
$$

Unfortunately, if either $u_{0} \neq 0$ or $f(0) \neq 0$, then here Lemma 3.2 (to be used with $\gamma=1$ ) gives only a first-order error bound, and such an order reduction is indeed observed numerically. In order to overcome this barrier we must modify the scheme by treating $u_{0}$ and $f_{0}=f(0)$ in a specific way.

To this end, we start from the solution formula (2.17). Setting $g=f-f(0)$, so that we can substitute $f=f_{0}+g$ with $g(0)=0$, and noting that

$$
\left(I+\partial^{-\alpha} A\right)^{-1}=I-\left(I+\partial^{-\alpha} A\right)^{-1} \partial^{-\alpha} A
$$

it turns out, assuming for the moment that $u_{0} \in D(A)$,

$$
u=u_{0}+\left(I+\partial^{-\alpha} A\right)^{-1}\left(-\partial^{-\alpha} A u_{0}+\partial^{-1} f_{0}+\partial^{-1} g\right) .
$$

Now it is natural to introduce the approximations defined by

$$
u^{h}=u_{0}+\left(I+\partial_{h}^{-\alpha} A\right)^{-1}\left(-\partial^{-\alpha} A u_{0}+\partial^{-1} f_{0}+\partial_{h}^{-1} g\right),
$$

i.e., by keeping the exact contributions

$$
\partial^{-\alpha} A u_{0}(t)=\frac{t^{\alpha}}{\Gamma(\alpha+1)} A u_{0}, \quad \partial^{-1} f_{0}(t)=t f_{0},
$$

instead of replacing them by $\partial_{h}^{-\alpha} A u_{0}$ and $\partial_{h}^{-1} f_{0}$, as we did in (4.1).

From a practical point of view, it is essential to implement (4.4) as a timestepping algorithm. After applying the operator $\left(I+\partial_{h}^{-\alpha} A\right)$ to both sides of (4.4) we get the equivalent formulation

$$
\left(I+\partial_{h}^{-\alpha} A\right)\left(u^{h}-u_{0}\right)=-\partial^{-\alpha} A u_{0}+\partial^{-1} f_{0}+\partial_{h}^{-1} g .
$$

For a function $v:[0,+\infty) \rightarrow X$ and real $\mu$, we have by definition

$$
\partial_{h}^{-\mu} v(t)=h^{\mu} \sum_{0 \leq j h \leq t} q_{j}^{(\mu)} v(t-j h), \quad t \geq 0
$$

where the quadrature weights $q_{j}^{(\mu)}, j \geq 0$, are provided by the expansion

$$
\delta(\zeta)^{-\mu}=\sum_{j=0}^{\infty} q_{j}^{(\mu)} \zeta^{j}
$$

or upon rewriting (3.3) as $\delta(\zeta)=\frac{3}{2}(1-\zeta)\left(1-\frac{1}{3} \zeta\right)$, by the explicit formula

$$
q_{j}^{(\mu)}=(-1)^{j}\left(\frac{2}{3}\right)^{\mu} \sum_{l=0}^{j} 3^{-l}\left(\begin{array}{c}
-\mu \\
j-l
\end{array}\right)\left(\begin{array}{c}
-\mu \\
l
\end{array}\right) .
$$


From these remarks, setting $t_{n}=n h, u_{n}=u^{h}\left(t_{n}\right), f_{n}=f\left(t_{n}\right), n \geq 0$, we see that at a nodal point $t_{n}>0$ the method (4.5) reads

$$
\begin{aligned}
u_{n}- & u_{0}+h^{\alpha} A \sum_{j=1}^{n} q_{n-j}^{(\alpha)}\left(u_{j}-u_{0}\right) \\
& =-\frac{t_{n}^{\alpha}}{\Gamma(\alpha+1)} A u_{0}+t_{n} f_{0}+h \sum_{j=1}^{n} q_{n-j}^{(1)}\left(f_{j}-f_{0}\right),
\end{aligned}
$$

which is an implicit equation for $u_{n}, n \geq 1$, once $u_{j}, 0 \leq j \leq n-1$, have been computed. The equation for $u_{n}$ is of the form

$$
\left(I+h^{\alpha} q_{0}^{(\alpha)} A\right) u_{n}=\xi+A \eta
$$

where $\xi, \eta \in X$. Since $q_{0}^{(\alpha)}=(2 / 3)^{\alpha}>0$, this equation possesses a unique solution, formally given by

$$
u_{n}=\left(I+h^{\alpha} q_{0}^{(\alpha)} A\right)^{-1} \xi+A\left(I+h^{\alpha} q_{0}^{(\alpha)} A\right)^{-1} \eta .
$$

This expression shows that $u_{n}$ depends continuously on $u_{0}$ and $f_{j}, 0 \leq j \leq n$. Actually, we deduce that the method is well defined even for $u_{0} \in X$, i.e., for initial data not in $D(A)$.

A direct implementation of $N$ time steps of the method requires the solution of $N$ linear systems, of $O\left(N^{2}\right)$ linear operations with vectors, and storing $O(N)$ vectors. In forthcoming work it is shown how this can be reduced to $O(N \log N)$ vector operations and to storing $O(\log N)$ vectors in active memory; cf. also [23] for a related fast convolution algorithm.

The method (4.7) can be rewritten in a form that makes the correction to (4.1) clearly visible:

$$
u_{n}+h^{\alpha} \sum_{j=1}^{n} q_{n-j}^{(\alpha)} A u_{j}+h^{\alpha} \widetilde{q}_{n}^{(\alpha)} A u_{0}=u_{0}+h \sum_{j=1}^{n} q_{n-j}^{(1)} f_{j}+h \widetilde{q}_{n}^{(1)} f_{0}
$$

with

$$
\widetilde{q}_{n}^{(\mu)}=\frac{n^{\mu}}{\Gamma(\mu+1)}-\sum_{j=1}^{n} q_{n-j}^{(\mu)} .
$$

The correction weights $\widetilde{q}_{n}^{(\mu)}$ for $\mu=\alpha$ and 1 can be interpreted as ensuring that the quadratures on both sides of (4.8) become exact for constant functions. By Lemma 3.2 for $K(s)=s^{-\mu}$ and $\gamma=1$, we have $h^{\mu} \widetilde{q}_{n}^{(\mu)}=O\left(t_{n}^{\mu-1} h\right)$. Moreover, for $t_{n}$ bounded away from 0 we have actually, by [21, Corollary 4.2], $h^{\mu} \widetilde{q}_{n}^{(\mu)}=$ $\frac{1}{2} h^{\mu} q_{n}^{(\mu)}+O\left(h^{2}\right)$. If we replace $h^{\mu} \widetilde{q}_{n}^{(\mu)}$ by $\frac{1}{2} h^{\mu} q_{n}^{(\mu)}$ for all $n$ in (4.8), we obtain a trapezoidal rule initial-point correction to (4.1) in the style of (3.9) (with $1_{h}(0)=\frac{1}{2}$ and $1_{h}(t)=1$ for $\left.t \geq h\right)$. For $t_{n}>0$ this is identical to the scheme that uses the approximation (3.9) in (4.3):

$$
u^{h}=u_{0}+\left(I+\partial_{h}^{-\alpha} A\right)^{-1}\left(-\partial_{h}^{-\alpha} A 1_{h} u_{0}+\partial_{h}^{-1} 1_{h} f_{0}+\partial_{h}^{-1} g\right) .
$$

With the second choice of $1_{h}$ mentioned in Section $3\left(1_{h}(0)=0,1_{h}(h)=\frac{3}{2}\right.$, $1_{h}(t)=1$ for $t \geq 2 h$ ), which yields $u^{h}(0)=u_{0}$, the approximation (4.9) is precisely the numerical method of [24]. Since for that particular choice of $1_{h}$ we have 
$1_{h}=\partial_{h} \partial^{-1} 1$ at grid-points $t_{n}$, the method of [24] can be written in a way that looks even closer to (4.4):

$$
u^{h}=u_{0}+\left(I+\partial_{h}^{-\alpha} A\right)^{-1}\left(-\partial_{h}^{-\alpha+1} \partial^{-1} A u_{0}+\partial^{-1} f_{0}+\partial_{h}^{-1} g\right) .
$$

While method (4.4) uses for $\left(I+\partial^{-\alpha} A\right)^{-1} \partial^{-\alpha} A u_{0}$ an approximation of the type (3.8) with $\nu=\alpha$, method (4.10) does the same with $\nu=1$. In view of their common general error bound (3.8), it would seem that both methods (4.4) and (4.10) share the same type of error behavior. However, this is not the case, and it turns out that (4.4) has superior approximation properties. While both methods have an $O\left(h^{2} / t_{n}^{2}\right)$ error in the case of general data $u_{0} \in X$ by (3.8), it will be seen that method (4.4) has, in contrast to (4.10), a uniform $O\left(h^{2}\right)$ error for regular data. The reason for this different behavior is that for method (4.4) we have, for $u_{0} \in D\left(A^{2}\right)$,

$$
\left(I+\partial_{h}^{-\alpha} A\right)^{-1} \partial^{-\alpha} A u_{0}=\partial^{-\alpha} A u_{0}-\left(I+\partial_{h}^{-\alpha} A\right)^{-1} \partial_{h}^{-\alpha} \partial^{-\alpha} A^{2} u_{0},
$$

where the first term on the right-hand side is exact, whereas for method (4.10) the analogous term $\partial_{h}^{-\alpha+1} \partial^{-1} A u_{0}$ has an error of $O\left(t^{\alpha-2} h^{2}\right)$ by (3.8), so that no uniform $O\left(h^{2}\right)$ error can be obtained for method (4.10). We therefore restrict our attention to method (4.4) in the following.

4.2. Error bounds. We have the following second-order error bound for method (4.4) or its equivalent formulations (4.7) and (4.8).

Theorem 4.1. Assume that $u_{0} \in X_{\nu}$ and $f_{0} \in X_{\sigma}$, for some $0 \leq \nu \leq 2$ and $0 \leq \sigma \leq 1$. Assume also that $f$ is twice differentiable and that $f^{\prime \prime} \in L^{1}((0,+\infty), X)$. Then, for each $T>0$, there exists $C=C(T)>0$ (independent of the data, the solution and the time step) such that, for $0<t_{n} \leq T$, there holds

$$
\left\|u_{n}-u\left(t_{n}\right)\right\| \leq C h^{2}\left(\frac{\left\|u_{0}\right\|_{\nu}}{t_{n}^{2-\alpha \nu}}+\frac{\left\|f_{0}\right\|_{\sigma}}{t_{n}^{1-\alpha \sigma}}+\left\|f^{\prime}(0)\right\|+\int_{0}^{t_{n}}\left\|f^{\prime \prime}(\tau)\right\| d \tau\right) .
$$

Proof. By linearity, it is enough to consider the three cases below.

(a) Case $u_{0} \in X_{\nu}$ and $f=0$. Subtracting (4.3) from (4.4) we get

$$
u^{h}-u=-\left(\left(I+\partial_{h}^{-\alpha} A\right)^{-1}-\left(I+\partial^{-\alpha} A\right)^{-1}\right) \partial^{-\alpha} A u_{0} .
$$

Suppose first that $u_{0} \in X$. Then we write

$$
u^{h}-u=\left(G\left(\partial_{h}\right)-G(\partial)\right) \partial^{-\alpha} u_{0},
$$

where

$$
G(s)=-A\left(I+s^{-\alpha} A\right)^{-1}, \quad|\arg (s)| \leq \theta / \alpha .
$$

Since clearly (see Lemma 2.2)

$$
\|G(s)\| \leq M|s|^{\alpha}, \quad|\arg (s)| \leq \theta / \alpha,
$$

Lemma 3.2 (with $\mu=-\alpha$ and $\gamma=\alpha+1$ ) yields

$$
\left\|u_{n}-u\left(t_{n}\right)\right\| \leq C(T) h^{2} t_{n}^{-2}\left\|u_{0}\right\|, \quad 0<t_{n} \leq T .
$$

Second, suppose that $u_{0} \in X_{2}$. Noting that

$$
-A\left(I+s^{-\alpha} A\right)^{-1}=-A+A^{2}\left(s^{\alpha}+A\right)^{-1},
$$

we obtain

$$
u^{h}-u=\left(G\left(\partial_{h}\right)-G(\partial)\right) \partial^{-\alpha} A^{2} u_{0},
$$

where now we adopt

$$
G(s)=\left(s^{\alpha}+A\right)^{-1}, \quad|\arg (s)| \leq \theta / \alpha .
$$


Since

$$
\|G(s)\| \leq M|s|^{-\alpha}, \quad|\arg (s)| \leq \theta / \alpha
$$

the application of Lemma 3.2 (with $\mu=\alpha$ and $\gamma=\alpha+1$ ) results in

$$
\left\|u_{n}-u\left(t_{n}\right)\right\| \leq C(T) h^{2} t_{n}^{2 \alpha-2}\left\|u_{0}\right\|_{2}, \quad 0<t_{n} \leq T .
$$

Finally, for $u_{0} \in X_{\nu}$, interpolation between (4.12) and (4.13) leads to

$$
\left\|u_{n}-u\left(t_{n}\right)\right\| \leq C(T) h^{2} t_{n}^{\nu \alpha-2}\left\|u_{0}\right\|_{\nu}, \quad 0<t_{n} \leq T .
$$

(b) Case $u_{0}=0, f=f_{0}$. Now we have

$$
u^{h}-u=\left(\left(I+\partial_{h}^{-\alpha} A\right)^{-1}-\left(I+\partial^{-\alpha} A\right)^{-1}\right) \partial^{-1} f_{0} .
$$

Therefore, for $f_{0} \in X_{\sigma}$, we can write

$$
u^{h}-u=\left(H_{\sigma}\left(\partial_{h}\right)-H_{\sigma}(\partial)\right) \partial^{-1} A^{\sigma} f_{0},
$$

where

$$
H_{\sigma}(s)=A^{1-\sigma}\left(s^{\alpha}+A\right)^{-1}, \quad|\arg (s)| \leq \theta / \alpha .
$$

By Lemma 2.2 we have

$$
\left\|H_{\sigma}(s)\right\| \leq M|s|^{-\alpha \sigma}, \quad|\arg (s)| \leq \theta / \alpha,
$$

hence, again by Lemma 3.2 (with $\mu=\alpha \sigma$ and $\gamma=2$ ), we get

$$
\left\|u_{n}-u\left(t_{n}\right)\right\| \leq C h^{2} t_{n}^{\alpha \sigma-1}\left\|f_{0}\right\|_{\sigma}, \quad 0<t_{n} \leq T .
$$

(c) Case $u_{0}=f_{0}=0$. In this case we have

$$
u^{h}-u=\left(K\left(\partial_{h}\right)-K(\partial)\right) g,
$$

where

$$
K(s)=s^{-1}\left(I+s^{-\alpha} A\right)^{-1}, \quad|\arg (s)| \leq \theta / \alpha .
$$

Since $g=t f^{\prime}(0)+t I * f^{\prime \prime}$, the relations (2.12) and (3.6) yield

$$
u^{h}-u=\left(K\left(\partial_{h}\right)-K(\partial)\right) t f^{\prime}(0)+\left(\left(K\left(\partial_{h}\right)-K(\partial)\right) t I\right) * f^{\prime \prime} .
$$

Since

$$
\|K(s)\| \leq M /|s|, \quad|\arg (s)| \leq \theta / \alpha,
$$

Lemma 3.2 (with $\mu=1$ and $\gamma=2$ ) implies that

$$
\left\|u_{n}-u\left(t_{n}\right)\right\| \leq C h^{2}\left(\left\|f^{\prime}(0)\right\|+\int_{0}^{t_{n}}\left\|f^{\prime \prime}(\tau)\right\| d \tau\right), \quad 0 \leq t_{n} \leq T .
$$

Taken together, these error bounds yield the stated result.

Theorem 4.1 provides error estimates under minimal regularity assumptions, in the style of the optimal estimates for parabolic problems $(\alpha=1)[6,15,16$. Thus, for $\nu=\sigma=0$, the situation referred to as the one with bad initial data, the error estimate reads $O\left(h^{2} / t_{n}^{2}\right)$. On the other hand, for $\nu=2 / \alpha$ and $\sigma=1 / \alpha$, we have guaranteed an error estimate of the form $O\left(h^{2}\right)$. It is noteworthy that, since $1<\alpha<2$, this optimal estimate holds with less regularity than the one required for parabolic problems [6, 15, 16]. Larger values of $\nu$ and $\sigma$ cannot improve the order but can improve the error constant.

With the semilinear problem in mind (see next section), we also need error estimates in intermediate spaces $X_{\rho}, 0 \leq \alpha \rho<1$. The following theorem focusses on the situation $u_{0} \in X_{2 / \alpha}$ and $f_{0} \in X_{1 / \alpha}$, which is the one encountered in the main result (Theorem 6.2) for the semilinear problem. 
Theorem 4.2. Assume that $u_{0} \in X_{2 / \alpha}, f_{0} \in X_{1 / \alpha}$, and that $f$ is twice differentiable in $X$, with $f^{\prime \prime} \in L^{1}((0,+\infty), X)$. Let $\rho \geq 0$, with $\alpha \rho<1$. Then, for each $T>0$ there exists $C=C(T)>0$ (independent of the solution, the data and the step size) such that, for $0<t_{n} \leq T$, there holds

$$
\left\|u_{n}-u\left(t_{n}\right)\right\|_{\rho} \leq \frac{C h^{2}}{t_{n}^{\alpha \rho}}\left(\left\|u_{0}\right\|_{2 / \alpha}+\left\|f_{0}\right\|_{1 / \alpha}+\left\|f^{\prime}(0)\right\|+\int_{0}^{t_{n}}\left\|f^{\prime \prime}(\tau)\right\| d \tau\right) .
$$

Proof. As in the proof of Theorem 4.1, we split the error into the three cases (a), (b) and (c) considered there. We realize that for cases (a) and (b) all we have to do is to shift the scale of the intermediate spaces, i.e., to adopt as phase space $\widetilde{X}=X_{\rho}$. Since

$$
X_{2 / \alpha}=\tilde{X}_{2 / \alpha-\rho}, \quad X_{1 / \alpha}=\tilde{X}_{1 / \alpha-\rho},
$$

Theorem 4.1, with $\nu=2 / \alpha-\rho$ and $\sigma=1 / \alpha-\rho$, readily shows (4.15) when $f=f_{0}$.

For the remaining case (c) we write $u^{h}-u$ again as in (4.14). Since by Lemma 2.2 ,

$$
\|K(s)\|_{X \rightarrow X_{\rho}} \leq M|s|^{\alpha \rho-1}, \quad|\arg (s)| \leq \theta / \alpha,
$$

we can apply Lemma 3.2 with $\mu=1-\alpha \rho$ and $\gamma=2$, which results in the desired estimate 4.15) for case (c).

4.3. Discrete propagators and stability. We introduce the discrete propagators of the numerical method, which will turn out to be very useful for the analysis of semilinear problems. We already know that $u_{n}$ depends linearly and boundedly on $u_{0}$ and $f_{j}, 0 \leq j \leq n$. In view of (4.4), there exist linear and bounded operators $P_{n}, Q_{n}, R_{n}: X \rightarrow X, n \geq 0$, such that

$$
u_{n}=P_{n} u_{0}+Q_{n} f_{0}+h \sum_{j=1}^{n} R_{n-j}\left(f_{j}-f_{0}\right) \text {. }
$$

We have the following stability bounds.

Lemma 4.3. For $0 \leq \alpha \rho<1$, the discrete propagators are bounded by

$$
\left\|P_{n}\right\|_{X_{\rho} \rightarrow X_{\rho}} \leq B, \quad\left\|Q_{n}\right\|_{X \rightarrow X_{\rho}} \leq B t_{n+1}^{1-\alpha \rho}, \quad\left\|R_{n}\right\|_{X \rightarrow X_{\rho}} \leq B t_{n+1}^{-\alpha \rho},
$$

where $B$ is independent of $n$ and $h$ with $n h \leq T$ for any fixed $T<\infty$.

The bound for $R_{n}$ shows that $\left\|R_{n}\right\|_{X \rightarrow X_{\rho}}$ behaves like a weakly singular function. This bound will become essential for the stability of the numerical method in the semilinear problem.

Proof. From (4.4) we have

$$
P_{n}=I-A\left(I+\partial_{h}^{-\alpha} A\right)^{-1} \frac{t^{\alpha}}{\Gamma(\alpha+1)} I\left(t_{n}\right), \quad Q_{n}=\left(I+\partial_{h}^{-\alpha} A\right)^{-1} t I\left(t_{n}\right) .
$$

We write

$$
\begin{aligned}
I-P_{n}= & \left(A\left(I+\partial_{h}^{-\alpha} A\right)^{-1} \frac{t^{\alpha}}{\Gamma(\alpha+1)} I\left(t_{n}\right)-A\left(I+\partial^{-\alpha} A\right)^{-1} \frac{t^{\alpha}}{\Gamma(\alpha+1)} I\left(t_{n}\right)\right) \\
& +A\left(I+\partial^{-\alpha} A\right)^{-1} \partial^{-\alpha} I\left(t_{n}\right) .
\end{aligned}
$$

For the difference on the right-hand side, Lemma 2.2 yields that we are, with $A\left(I+s^{-\alpha} A\right)^{-1}$ in the role of $K(s)$, in the situation of Lemma 3.2 with $\mu=-\alpha$ and $\gamma=\alpha+1$, which yields a bound of $O\left(t_{n}^{-2} h^{2}\right)$ for the norm of the difference. The last term in $I-P_{n}$ is the inverse Laplace transform of $s^{-1} s^{-\alpha} A\left(I+s^{-\alpha} A\right)^{-1}$, and 
hence, by Lemma 2.1 with $\mu=1$, this term is bounded uniformly in $t_{n}$. This gives the bound for $P_{n}$.

Similarly, for $Q_{n}$, Lemma 2.2 with $\nu=\rho$ shows that we are in the situation of Lemma 3.2 with $\mu=-\alpha \rho$ and $\gamma=2$, and of Lemma 2.1 with $\mu=2-\alpha \rho$. This yields the stated bound for $Q_{n}$.

The operators $h R_{n}, n \geq 0$, are the convolution quadrature weights corresponding to the Laplace transform $K(s)=s^{-1}\left(I+s^{-\alpha} A\right)^{-1}$ used in the proof of Theorems 4.1 and 4.2, that is, they are the coefficients in the series expansion

$$
h \sum_{n=0}^{\infty} R_{n} \zeta^{n}=(\delta(\zeta) / h)^{-1}\left(I+(\delta(\zeta) / h)^{-\alpha} A\right)^{-1} .
$$

Using (4.16) and Lemma 3.1 with $\mu=1-\alpha \rho$, we deduce the bounds for $R_{n}$.

In addition to stability with respect to perturbations in the data $u_{0}$ and $f\left(t_{n}\right)$ as expressed by Lemma 4.3. it is of interest to study the effect of perturbations that result from an inexact solution of the linear system of equations in (4.8). Supposing that the linear system in the $n$-th step is solved only up to a defect $\delta_{n}$, so that we compute perturbed values $\widetilde{u}_{n}$, the equation for the errors $\varepsilon_{n}=\widetilde{u}_{n}-u_{n}$ becomes

$$
\varepsilon_{n}+h^{\alpha} \sum_{j=1}^{n} q_{n-j}^{(\alpha)} A \varepsilon_{j}=\delta_{n}
$$

or equivalently, with $\varepsilon^{h}=\left(\varepsilon_{n}\right)$ and $\delta^{h}=\left(\delta_{n}\right)$,

$$
\left(I+\partial_{h}^{-\alpha} A\right) \varepsilon^{h}=\delta^{h}, \quad \text { or } \quad \varepsilon^{h}=\left(I+\partial_{h}^{-\alpha} A\right)^{-1} \delta^{h} .
$$

By Lemma 3.1 (with $\mu=0$ ) for $K(s)=\left(I+s^{-\alpha} A\right)^{-1}$ and by Lemma 2.2 (with $\nu=0)$ we thus have

$$
\varepsilon_{n}=h \sum_{j=1}^{n} \kappa_{n-j} \delta_{j} \quad \text { with } \quad\left\|\kappa_{n}\right\| \leq B(n h)^{-1} .
$$

Hence we obtain the stability bound

$$
\max _{j=1, \ldots, n}\left\|\varepsilon_{j}\right\| \leq B \log (n+1) \max _{j=1, \ldots, n}\left\|\delta_{j}\right\|, \quad n \geq 1 .
$$

\section{The Semilinear PRoblem}

We now consider the semilinear initial value problem

$$
u^{\prime}(t)+\frac{1}{\Gamma(\beta)} \int_{0}^{t}(t-\tau)^{\beta-1} A u(\tau) d \tau=F(t, u(t)), \quad 0<t \leq T, \quad u(0)=u_{0},
$$

where again $0<\beta<1, A$ is a sectorial operator satisfying (2.2) with (2.3), and now there is the nonlinearity

$$
F:[0, T] \times X_{\rho} \rightarrow X \quad \text { with } \quad \alpha \rho<1,
$$

again for $\alpha=\beta+1$. For convenience we assume that $F$ is globally Lipschitz continuous, in the sense that there exists $L>0$ such that

$$
\|F(t, v)-F(t, w)\| \leq L\|v-w\|_{\rho}, \quad 0 \leq t \leq T, \quad v, w \in X_{\rho} .
$$

This assumption could be replaced by a local Lipschitz condition on every ball of $X_{\rho}$ without inflicting essential changes in the subsequent results and in their proofs, apart from the existence of the (mild) solution on the whole interval $[0, T]$, which is guaranteed in the globally Lipschitz situation and has to be assumed a priori otherwise. 
As in (2.4) we have the integrated equation

$$
u(t)+\partial^{-\alpha} A u(t)=u_{0}+\int_{0}^{t} F(\tau, u(\tau)) d \tau .
$$

A mild solution of (5.1) or (5.2) is a solution $u \in C\left([0, T], X_{\rho}\right)$ of the equation

$$
u=\left(I+\partial^{-\alpha} A\right)^{-1}\left(u_{0}+\partial^{-1} F(\cdot, u)\right) \text {. }
$$

This can be rewritten as the Volterra integral equation

$$
u(t)=k(t) u_{0}+\int_{0}^{t} k(t-\tau) F(\tau, u(\tau)) d \tau,
$$

where $k(t)$ is the inverse Laplace transform of $K(s)=s^{-1}\left(I+s^{-\alpha} A\right)^{-1}$, which by Lemmas 2.2 and 2.1 is bounded by

$$
\|k(t)\|_{X_{\rho} \rightarrow X_{\rho}} \leq B, \quad\|k(t)\|_{X \rightarrow X_{\rho}} \leq B t^{-\alpha \rho}, \quad t>0 .
$$

By the standard Picard iteration argument, this weakly singular Volterra integral equation has a unique solution $u \in C\left([0, T], X_{\rho}\right)$ for initial data $u_{0} \in X_{\rho}$. The following regularity result extends Lemma 2.3 to the semilinear equation and will be proved in Section 7 .

Theorem 5.1. Assume that $F:[0, T] \times X_{\rho} \rightarrow X$ with $\alpha \rho<1$ is Lipschitz bounded and twice continuously differentiable. Suppose $u_{0} \in X_{2 / \alpha}$ and $F\left(0, u_{0}\right) \in X_{1 / \alpha}$. Then the semilinear problem (5.1) has a unique mild solution, of the form

$$
u(t)=u_{0}+t F\left(0, u_{0}\right)-\frac{t^{\alpha}}{\Gamma(\alpha+1)} A u_{0}+v(t),
$$

where $v \in C^{2}([0, T], X)$ with $v(0)=v^{\prime}(0)=0$, and $u \in C^{2}\left((0, T], X_{\rho}\right)$ with

$$
\left\|u^{\prime \prime}(t)\right\|_{\rho} \leq C t^{-r}, \quad \text { for } \quad r=\max (\alpha \rho, 2-\alpha)<1 .
$$

As a direct consequence of Theorem 5.1, we note the following for further use.

Corollary 5.2. Under the conditions of Theorem 5.1, the function $f(t)=F(t, u(t))$ is in $C^{2}((0, T], X)$ with $\left\|f^{\prime \prime}(t)\right\| \leq C t^{-r}$, where $r<1$.

\section{Time Discretization of THE SEMilinear EQUATION}

6.1. The numerical method. In this section we adapt the numerical scheme (4.4) to approximate the solution $u:[0, T] \rightarrow X_{\rho}, 0 \leq \alpha \rho<1$, of the semilinear problem (5.2), by letting

$$
u^{h}=u_{0}+\left(I+\partial_{h}^{-\alpha} A\right)^{-1}\left(-\partial^{-\alpha} A u_{0}+\partial^{-1} f_{0}+\partial_{h}^{-1}\left(F\left(\cdot, u^{h}\right)-f_{0}\right)\right),
$$

where $f_{0}$ stands for $F\left(0, u_{0}\right)$. In view of (4.7), we are thus led to consider the approximations $u_{n}$ to $u\left(t_{n}\right), 0 \leq t_{n} \leq T$, defined recursively by $u_{0}$ as given and, for $n \geq 1$,

$$
\begin{aligned}
u_{n}- & u_{0}+h^{\alpha} A \sum_{j=1}^{n} q_{n-j}^{(\alpha)}\left(u_{j}-u_{0}\right) \\
& =-\frac{t_{n}^{\alpha}}{\Gamma(\alpha+1)} A u_{0}+t_{n} f_{0}+h \sum_{j=1}^{n} q_{n-j}^{(1)}\left(F\left(t_{j}, u_{j}\right)-f_{0}\right)
\end{aligned}
$$

where the quadrature weights $q_{j}^{(\mu)}$ are given in (4.6). 
6.2. Solvability of the discrete equations. The equation for $u_{n}$ reads

$$
\left(I+h^{\alpha} q_{0}^{(\alpha)} A\right) u_{n}=\xi+A \eta+h q_{0}^{(1)} F\left(t_{n}, u_{n}\right),
$$

for certain $\xi \in X$ and $\eta \in X_{\rho}$. The solvability of (6.3) is covered by the following lemma. Recall that

$$
\left\|(I+\lambda A)^{-1}\right\|_{X \rightarrow X_{\rho}} \leq M / \lambda^{1-\rho}, \quad \lambda>0 .
$$

Lemma 6.1. Assume that $\xi \in X, \eta \in X_{\rho}$, and that

$$
h^{1-\alpha \rho} M L q_{0}^{(1)} /\left(q_{0}^{(\alpha)}\right)^{\rho}<1 .
$$

Then equation (6.3) admits a unique solution $u_{n} \in X_{\rho}$, and $u_{n}$ depends continuously on $\xi \in X$ and $\eta \in X_{\rho}$.

Proof. The solvability of $\underline{6.3}$ is equivalent to the existence of a fixed point for the mapping $S: X_{\rho} \rightarrow X_{\rho}$ defined by

$$
S(u)=\left(I+h^{\alpha} q_{0}^{(\alpha)} A\right)^{-1}\left(\xi+A \eta+h q_{0}^{(1)} F\left(t_{n}, u\right)\right) .
$$

Note that $\left(I+h^{\alpha} q_{0}^{(\alpha)} A\right)^{-1}$ maps $X$ into $X_{\rho}$ and that, since

$$
\left(I+h^{\alpha} q_{0}^{(\alpha)} A\right)^{-1} A=\frac{1}{h^{\alpha} q_{0}^{(\alpha)}}\left(\left(I+h^{\alpha} q_{0}^{(\alpha)} A\right)^{-1}-I\right),
$$

certainly $S$ maps $X_{\rho}$ to $X_{\rho}$. Moreover, because of (6.4), we have

$$
\left\|\left(I+h^{\alpha} q_{0}^{(\alpha)} A\right)^{-1}\right\|_{X \rightarrow X_{\rho}} \leq \frac{M}{h^{\alpha \rho}\left(q_{0}^{(\alpha)}\right)^{\rho}},
$$

so that the restriction (6.5) implies that $S$ is a contraction.

6.3. Error bounds. Here we obtain the main result of the paper.

Theorem 6.2. Assume that $F:[0, T] \times X_{\rho} \rightarrow X$ with $\alpha \rho<1$ is Lipschitz bounded and twice continuously differentiable. Suppose $u_{0} \in X_{2 / \alpha}$ and $F\left(0, u_{0}\right) \in X_{1 / \alpha}$. Then there exist $h_{0}>0$ and $C>0$ such that, for $0<h \leq h_{0}$, the numerical approximations $u_{n}, 0<t_{n} \leq T$, given by (6.2) are uniquely defined, and their errors are bounded by

$$
\begin{aligned}
& \left\|u_{n}-u\left(t_{n}\right)\right\|_{\rho} \leq C h^{2} t_{n}^{-\alpha \rho}, \\
& \left\|u_{n}-u\left(t_{n}\right)\right\| \leq C h^{2} .
\end{aligned}
$$

Proof. Select $h_{0}>0$ fulfilling the threshold condition (6.5). Then, for $0<h \leq h_{0}$, the numerical solutions $u_{n} \in X_{\rho}, 0 \leq t_{n} \leq T$, are well defined. Moreover, obviously $u:[0, T] \rightarrow X_{\rho}$ is the solution of the auxiliary problem

$$
u(t)+\partial^{-\alpha} A u(t)=u_{0}+\partial^{-1} f(t), \quad 0 \leq t \leq T,
$$

where $f(t)=F(t, u(t)), 0 \leq t \leq T$.

Let $v_{n}, 0 \leq t_{n} \leq T$, denote the approximations to $u$ obtained by applying the method (4.7) to problem (6.8). In view of the imposed regularity $u_{0} \in X_{2 / \alpha}$ and $f_{0} \in X_{1 / \alpha}$ and of the regularity of $f(t)$ given by Corollary [5.2, we can apply Theorem 4.2 to obtain that there exists $C>0$ such that

$$
\left\|v_{n}-u\left(t_{n}\right)\right\|_{\rho} \leq C h^{2} t_{n}^{-\alpha \rho}, \quad 0 \leq t_{n} \leq T
$$


and Theorem 4.1 (with $\nu=2 / \alpha$ and $\sigma=1 / \alpha$ ) gives

$$
\left\|v_{n}-u\left(t_{n}\right)\right\| \leq C h^{2}, \quad 0 \leq t_{n} \leq T .
$$

On the other hand, expressing $v_{n}$ in terms of the data, through the discrete propagators as in (4.17), leads to

$$
v_{n}=P_{n} u_{0}+Q_{n} f_{0}+h \sum_{j=1}^{n} R_{n-j}\left(f\left(t_{j}\right)-f_{0}\right), \quad 0<t_{n} \leq T .
$$

Analogously, we can express

$$
u_{n}=P_{n} u_{0}+Q_{n} f_{0}+h \sum_{j=1}^{n} R_{n-j}\left(F\left(t_{j}, u_{j}\right)-f_{0}\right), \quad 0<t_{n} \leq T .
$$

Therefore, for $0<t_{n} \leq T$, we get

$$
\begin{aligned}
u_{n}-u\left(t_{n}\right) & =v_{n}-u\left(t_{n}\right)+u_{n}-v_{n} \\
& =v_{n}-u\left(t_{n}\right)+h \sum_{j=1}^{n} R_{n-j}\left(F\left(t_{j}, u_{j}\right)-F\left(t_{j}, u\left(t_{j}\right)\right)\right),
\end{aligned}
$$

which, by Lemma 4.3, implies that

$$
\left\|u_{n}-u\left(t_{n}\right)\right\|_{\rho} \leq\left\|v_{n}-u\left(t_{n}\right)\right\|_{\rho}+h L B \sum_{j=1}^{n} t_{n-j+1}^{-\alpha \rho}\left\|u_{j}-u\left(t_{j}\right)\right\|_{\rho} .
$$

After inserting (6.9), Gronwall's lemma for weakly singular kernels (see, e.g., Lemma 2.1 in [11] or Lemma 6 in [5]) readily shows (6.6). In the same way, taking the norm in $X$, we have

$$
\left\|u_{n}-u\left(t_{n}\right)\right\| \leq\left\|v_{n}-u\left(t_{n}\right)\right\|+h L B \sum_{j=1}^{n}\left\|u_{j}-u\left(t_{j}\right)\right\|_{\rho} .
$$

Hence, by using (6.10) and (6.6), we finally get (6.7).

\section{Regularity of SOlutions}

In this section we prove Lemma 2.3 and Theorem 5.1 We first give the proof of regularity of the linear problem.

Proof of Lemma 2.3. We split the (distributional) second derivative of $u$ as

$$
\begin{aligned}
\partial^{2} u= & \partial^{2}\left(I+\partial^{-\alpha} A\right)^{-1} u_{0}+\partial\left(I+\partial^{-\alpha} A\right)^{-1} f(0) \\
& +\left(I+\partial^{-\alpha} A\right)^{-1} f^{\prime}(0)+\partial^{-1}\left(I+\partial^{-\alpha} A\right)^{-1} \partial^{2}\left(f-f(0)-t f^{\prime}(0)\right) \\
\equiv & w_{1}+w_{2}+w_{3}+w_{4} .
\end{aligned}
$$

The first term, $w_{1}(t)$, is the inverse Laplace transform of

$$
\begin{aligned}
W_{1}(s)= & s^{2}\left(I+s^{-\alpha} A\right)^{-1} \frac{u_{0}}{s} \\
= & s\left(I+s^{-\alpha} A\right)^{-1} A^{-2 / \alpha} \cdot A^{2 / \alpha} u_{0} \\
= & s\left(\left(I+s^{-\alpha} A\right)^{-1} A^{-2 / \alpha}-A^{-2 / \alpha}+s^{-\alpha} A^{1-2 / \alpha}\right) A^{2 / \alpha} u_{0} \\
& +s u_{0}-s^{1-\alpha} A u_{0} .
\end{aligned}
$$


By Lemma 2.2. (2.15) with $\nu=-2 / \alpha$, we have the bound

$$
\left\|s\left(\left(I+s^{-\alpha} A\right)^{-1} A^{-2 / \alpha}-A^{-2 / \alpha}+s^{-\alpha} A^{1-2 / \alpha}\right) A^{2 / \alpha} u_{0}\right\| \leq C|s|^{-1}\left\|u_{0}\right\|_{2 / \alpha} .
$$

By Lemma 2.1 with $\mu=1$, this is the Laplace transform of a function bounded by $C\left\|u_{0}\right\|_{2 / \alpha}$. The second and third terms in $W_{1}(s)$ are the Laplace transforms of $\delta_{0}^{\prime} u_{0}$ (with $\delta_{0}^{\prime}$ the derivative of Dirac's delta) and $-\frac{t^{\alpha-2}}{\Gamma(\alpha-1)} A u_{0}$, respectively. Hence we have

$$
\left\|w_{1}(t)+\frac{t^{\alpha-2}}{\Gamma(\alpha-1)} A u_{0}\right\| \leq C\left\|u_{0}\right\|_{2 / \alpha}, \quad t>0 .
$$

Similarly, $w_{2}(t)$ is the inverse Laplace transform of

$$
W_{2}(s)=\left(\left(I+s^{-\alpha} A\right)^{-1} A^{-1 / \alpha}-A^{-1 / \alpha}\right) A^{1 / \alpha} f(0)+f(0),
$$

which by Lemma 2.2, (2.14) with $\nu=-1 / \alpha$, is bounded by

$$
\left\|W_{2}(s)-f(0)\right\| \leq C|s|^{-1}\|f(0)\|_{1 / \alpha} .
$$

Since $f(0)$ is the inverse Laplace transform of $\delta_{0} f(0)$, Lemma 2.1 with $\mu=1$ implies

$$
\left\|w_{2}(t)\right\| \leq C\|f(0)\|_{1 / \alpha}, \quad t>0 .
$$

The same argument applied to $W_{3}(s)=s^{-1}\left(I+s^{-\alpha} A\right)^{-1} f^{\prime}(0)$ yields

$$
\left\|w_{3}(t)\right\| \leq C\left\|f^{\prime}(0)\right\| \text {. }
$$

Finally, $w_{4}$ is the convolution $K(\partial) f^{\prime \prime}=k * f^{\prime \prime}$, where $K(s)=s^{-1}\left(I+s^{-\alpha} A\right)^{-1}$ is bounded in operator norm by

$$
\|K(s)\| \leq C|s|^{-1}
$$

so that, again by Lemma (2.1) with $\mu=1$, its inverse Laplace transform $k(t)$ is bounded by

$$
\|k(t)\| \leq C .
$$

This implies that $w_{4}=k * f^{\prime \prime}$ is bounded by

$$
\left\|w_{4}(t)\right\|=\left\|\int_{0}^{t} k(t-\tau) f^{\prime \prime}(\tau) d \tau\right\| \leq C \int_{0}^{t}\left\|f^{\prime \prime}(\tau)\right\| d \tau
$$

Putting the above pieces together gives the continuity of $v^{\prime \prime}(t)$ on $[0, T]$ and a bound in the $X$-norm,

$$
\left\|v^{\prime \prime}(t)\right\| \leq C\left(\left\|u_{0}\right\|_{2 / \alpha}+\|f(0)\|_{1 / \alpha}+\left\|f^{\prime}(0)\right\|+\int_{0}^{t}\left\|f^{\prime \prime}(\tau)\right\| d \tau\right) .
$$

The result in $X_{\rho}$ is obtained by the same arguments as long as $\alpha \rho \leq 2-\alpha$. We now use Lemma 2.2 with $\nu=\rho-2 / \alpha, \rho-1 / \alpha, \rho$. This yields a situation to which Lemma 2.1 applies with $\mu=1-\alpha \rho>0$ in each of the cases. The estimate now becomes

$$
\left\|v^{\prime \prime}(t)\right\|_{\rho} \leq \frac{C}{t^{\alpha \rho}}\left(\left\|u_{0}\right\|_{2 / \alpha}+\|f(0)\|_{1 / \alpha}+\left\|f^{\prime}(0)\right\|+t \max _{0 \leq \tau \leq t}\left\|f^{\prime \prime}(\tau)\right\|\right) .
$$

For $\alpha \rho>2-\alpha$ we need to treat the term with $u_{0}$ differently. We then write

$$
\begin{aligned}
A^{\rho} W_{1}(s) & =s^{2} A^{\rho}\left(I+s^{-\alpha} A\right)^{-1} \frac{u_{0}}{s} \\
& =s\left(I+s^{-\alpha} A\right)^{-1} A^{\rho-2 / \alpha} \cdot A^{2 / \alpha} u_{0} \\
& =s\left(\left(I+s^{-\alpha} A\right)^{-1} A^{\rho-2 / \alpha}-A^{\rho-2 / \alpha}\right) A^{2 / \alpha} u_{0}+s A^{\rho} u_{0} .
\end{aligned}
$$


Since here $-1 \leq \rho-2 / \alpha \leq 0$, we now use the second bound of Lemma 2.2 to conclude

$$
\left\|s\left(\left(I+s^{-\alpha} A\right)^{-1} A^{\rho-2 / \alpha}-A^{\rho-2 / \alpha}\right) A^{2 / \alpha} u_{0}\right\| \leq C|s|^{-1+\rho \alpha}\left\|u_{0}\right\|_{2 / \alpha} .
$$

By Lemma 2.1 with $\mu=1-\rho \alpha$ we thus obtain

$$
\left\|w_{1}(t)\right\|_{\rho} \leq \frac{C}{t^{\alpha \rho}}\left\|u_{0}\right\|_{2 / \alpha}
$$

and the result follows as before.

Proof of Theorem 5.1. (a) We compare the solution $u$ of (5.2) with the solution to the linear problem with initial value $u_{0}$ and the constant inhomogeneity $F\left(0, u_{0}\right)$,

$$
w+\partial^{-\alpha} A w=u_{0}+\partial^{-1} F\left(0, u_{0}\right)
$$

the solution of which is of the form stated in the theorem, by Lemma 2.3. We therefore make the ansatz $u=w+x$ and begin by giving an equation for $x$. Setting

$$
G(t, \xi)=F(t, w(t)+\xi)-F\left(0, u_{0}\right)
$$

(note that $w(t) \in X_{\rho}$ with an integrable second derivative in $X_{\rho}$ ), we obtain that $x$ must satisfy the equation

$$
x=\left(I+\partial^{-\alpha} A\right)^{-1} \partial^{-1} G(\cdot, x) .
$$

By Lemma 2.2, $K(s)=\left(I+s^{-\alpha} A\right)^{-1} s^{-1}: X_{\rho} \rightarrow X$ is bounded by

$$
\|K(s)\|_{X \rightarrow X_{\rho}} \leq M|s|^{\alpha \rho-1}
$$

so that by Lemma 2.1. $K(s)$ is the Laplace transform of a kernel $k(t)$ bounded by

$$
\|k(t)\|_{X \rightarrow X_{\rho}} \leq C t^{-\alpha \rho} .
$$

The equation for $x$ is thus a Volterra integral equation with weakly singular kernel and Lipschitz nonlinearity,

$$
x(t)=\int_{0}^{t} k(t-\tau) G(\tau, x(\tau)) d \tau,
$$

which has a unique solution $x \in C\left([0, T], X_{\rho}\right)$.

(b) If $x$ were in $C^{1}\left([0, T], X_{\rho}\right)$, then its derivative would solve the linear Volterra integral equation

$$
y(t)=\int_{0}^{t} k(t-\tau)\left(G_{t}(\tau, x(\tau))+G_{x}(\tau, x(\tau)) y(\tau)\right) d \tau,
$$

which has a unique solution $y \in C\left([0, T], X_{\rho}\right)$. It remains to show that indeed $y=x^{\prime}$. For this purpose we consider, for small $\epsilon>0$, the regularized nonlinearity

$$
G_{\epsilon}=(I+\epsilon A)^{-1} G: X_{\rho} \rightarrow X_{1},
$$

which together with its partial derivatives converges to $G$ in the $X$ norm as $\epsilon \rightarrow 0$, uniformly on bounded sets of arguments $(t, \xi)$. We define $x_{\epsilon}$ as the solution of the modified Volterra integral equation

$$
x_{\epsilon}(t)=\int_{0}^{t} k(t-\tau) G_{\epsilon}\left(\tau, x_{\epsilon}(\tau)\right) d \tau,
$$

and with a Gronwall inequality we obtain

$$
x_{\epsilon} \rightarrow x \quad \text { in } C\left([0, T], X_{\rho}\right) .
$$


The point of this construction is now that $x_{\epsilon}$ is continuously differentiable, since its distributional derivative is given by

$$
x_{\epsilon}^{\prime}=\left(I+\partial^{-\alpha} A\right)^{-1} G_{\epsilon}\left(\cdot, x_{\epsilon}\right)=G_{\epsilon}\left(\cdot, x_{\epsilon}\right)-\left(I+\partial^{-\alpha} A\right)^{-1} \partial^{-\alpha} A G_{\epsilon}\left(\cdot, x_{\epsilon}\right),
$$

which is in $C\left([0, T], X_{\rho}\right)$ because $\left(I+s^{-\alpha} A\right)^{-1} s^{-\alpha}$ is, by Lemmas 2.2 and 2.1, the Laplace transform of a locally integrable kernel mapping from $X$ to $X_{\rho}$. Upon differentiating the integral equation for $x_{\epsilon}$ we then obtain, in $X_{\rho}$,

$$
x_{\epsilon}^{\prime}(t)=\int_{0}^{t} k(t-\tau)\left(G_{\epsilon, t}(\tau, x(\tau))+G_{\epsilon, x}\left(\tau, x_{\epsilon}(\tau)\right) x_{\epsilon}^{\prime}(\tau)\right) d \tau .
$$

Comparing this with the equation for $y$ and using a Gronwall inequality we obtain

$$
x_{\epsilon}^{\prime} \rightarrow y \quad \text { in } C\left([0, T], X_{\rho}\right) .
$$

Since differentiation is a closed operator on $C\left([0, T], X_{\rho}\right)$, it follows that $x \in$ $C^{1}\left([0, T], X_{\rho}\right)$ and $x^{\prime}=y$.

(c) We proceed similarly for the second derivative of $x$, which (formally) solves the linear Volterra integral equation

$z(t)=\int_{0}^{t} k(t-\tau)\left(G_{t t}+2 G_{t x} x^{\prime}+G_{x x}\left(x^{\prime}, x^{\prime}\right)+G_{x} z\right)(\tau) d \tau+k(t)\left(G_{t}+G_{x} x^{\prime}\right)(0)$.

Since $\left\|G_{t t}\right\| \leq C t^{\alpha-2}$ because of the presence of the second derivative of the term $\left(t^{\alpha} / \Gamma(\alpha+1)\right) A u_{0}$ in $w^{\prime \prime}(t)$, this integral equation for $z$ is of the form

$$
z(t)=g(t)+\int_{0}^{t} k(t-\tau) G_{x}(\tau, x(\tau)) z(\tau) d \tau
$$

where $g$, and hence also $z$, satisfies $g \in C\left((0, T], X_{\rho}\right)$ with $\|g(t)\|_{\rho} \leq C t^{\alpha-1-\alpha \rho} \leq$ $C t^{-\alpha \rho}$. As in part (b), it is shown that $z$ is indeed the derivative of $y=x^{\prime}$. This proves the result for $u=w+x$.

\section{The SUbDifFUsion EQUATION}

In this section we extend our results to equation (2.4), viz.,

$$
u+\partial^{-\alpha} A u=u_{0}+\partial^{-1} f,
$$

in the situation of

$$
0<\alpha<1
$$

This equation has recently received much attention in the physical literature as a model equation for slow anomalous diffusion; see [28] and references therein. The techniques of this paper apply equally to this situation, with slight modifications in the results. The resolvent bounds of Lemma 2.2 remain unchanged for $0<\alpha<1$, and the regularity result of Lemma 2.3 now becomes the following: under the conditions of Lemma 2.3, the solution is of the form

$$
u(t)=\sum_{m, l \geq 0 ; m+l \alpha<2} c_{m l} t^{m+l \alpha}+v(t)
$$

with $c_{m l} \in X$ and $v \in C^{2}([0, T], X)$ with $v(0)=v^{\prime}(0)=0$. For equation (8.1) with $0<\alpha<1$ the numerical method (4.4), or equivalently (4.7), remains unchanged. 
However, the error bound of Theorem 4.1 no longer remains valid. Instead of (4.11) we now have for method (4.4) the weaker estimate, for $0 \leq \nu \leq 2$ and $0 \leq \sigma \leq 1$,

$$
\left\|u_{n}-u\left(t_{n}\right)\right\| \leq C h^{1+\alpha} \frac{\left\|u_{0}\right\|_{\nu}}{t_{n}^{1+\alpha-\alpha \nu}}+C h^{2}\left(\frac{\left\|f_{0}\right\|_{\sigma}}{t_{n}^{1-\alpha \sigma}}+\left\|f^{\prime}(0)\right\|+\int_{0}^{t_{n}}\left\|f^{\prime \prime}(\tau)\right\| d \tau\right)
$$

This is obtained with the same proof based on Lemma 3.2, which can be used only with $\gamma=1+\alpha<2$ in the term of (4.4) containing $u_{0}$.

On the other hand, method (4.10) here still admits a (non-uniform) second-order error bound: 4.11) holds for $0 \leq \nu \leq 1$ and $0 \leq \sigma \leq 1$. This is again obtained by using Lemma 3.2, now with $\gamma=2$.

For very regular data $u_{0}$ and $f$, which are such that in (8.2) $c_{m l} \in D(A)$ and $v \in C^{2}([0, T], D(A))$, a uniform second-order error bound can be obtained by discretizing (8.1) with the fractional BDF2 method with correction terms that integrate exactly all the powers appearing in the expansion terms in (8.2), as in [19, 20]. In a Hilbert space framework, the uniform $O\left(h^{2}\right)$ error bound then follows directly from a result of [20]. In the Banach space framework of this paper, such an error bound is obtained by an argument based on consistency (Lemma 3.2 for $\partial^{-\alpha} A u$ and $\partial^{-1} f$ ) and stability (Lemma 4.3, which also holds for $0<\alpha<1$ ).

\section{NumERICAL EXPERIMENTS}

We present several numerical experiments to illustrate the main results in the paper. For different problems (2.1) or (5.1), we select $T>0, N \geq 1$, and we apply the numerical method (4.7) or (6.2) with time step size $h=T / N$. In order to estimate the errors, we adopt as a reference solution the discrete one corresponding to $h_{e}=T / N_{e}$, where $N_{e} \gg N$. In the experiments, we will focus on the behavior of the error constants rather than on the errors themselves.

9.1. Experiment 1. We consider the scalar equation

$$
u(t)+\partial^{-\alpha} u(t)=1, \quad t \geq 0
$$

i.e., the linear, homogeneous problem with $A=1, X=\mathbf{C}$ and $u_{0}=1$. For $T=2$, we select $N=2^{k}, 4 \leq k \leq 9$, and $N_{e}=3200$.
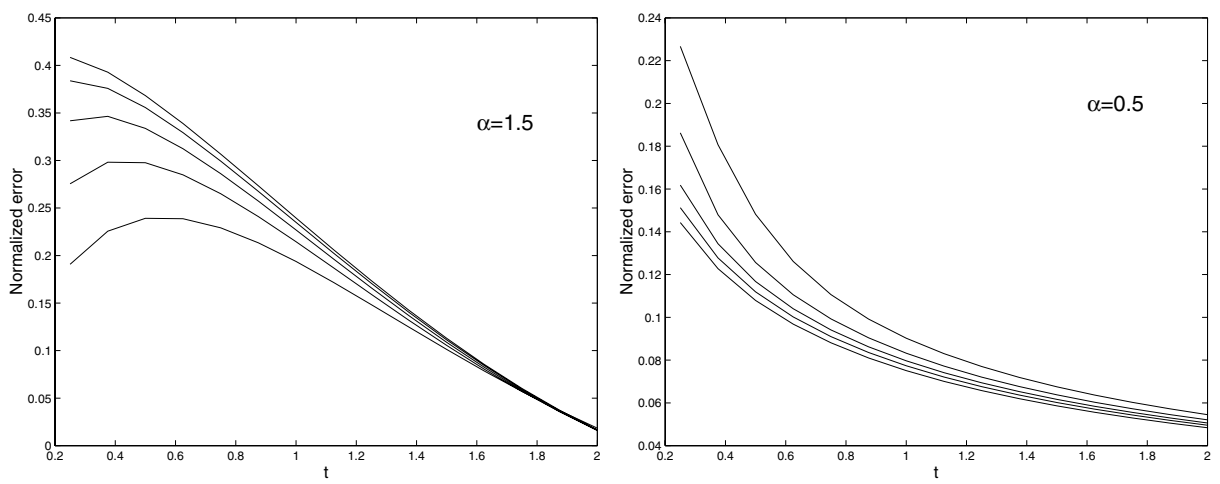

Figure 1. Left: Errors $/ h^{2}$ vs. $t$ for $\alpha=1.5$. Right: Errors $\cdot t^{1-\alpha} / h^{1+\alpha}$ vs. $t$ for $\alpha=0.5$. 
If $1 \leq \alpha<2$, we normalize the resulting errors by dividing them by the corresponding $h^{2}$. However, if $0<\alpha<1$, we normalize by multiplying the errors by $t^{1-\alpha} / h^{1+\alpha}$. In view of (4.15), with $\rho=0$, and (8.3), with $\nu=2$, what we expect is these normalized errors to remain bounded.

For $\alpha=1.5$ and $\alpha=0.5$ we plot the above normalized errors, for the different values of $h$ used, on the coarser grid with $N=16$. Figure 1 shows that the error constants behave in a coherent way. While the left-hand plot confirms a uniform $O\left(h^{2}\right)$ error for $\alpha=1.5$, the right-hand plot for $\alpha=0.5$ shows that in the subdiffusion case we do not have a uniform-in-time $O\left(h^{1+\alpha}\right)$ error bound for method (4.7).

9.2. Experiment 2. This and the next experiment are on the fractional Burgers' equation

$$
u(x, t)-\partial^{-\alpha} u_{x x}(x, t)=u_{0}-\partial^{-1}\left(u(x, t)^{2}\right)_{x}, \quad 0 \leq x \leq \pi, \quad t \geq 0,
$$

with homogeneous Dirichlet boundary conditions, for different $1 \leq \alpha<2$, and initial conditions $u_{0}(x)$ with different degrees of smoothness. As phase space we adopt $X=L_{p}(0, \pi), 1 \leq p<+\infty$, or $X=C[0, \pi]$, if $p=+\infty$. The underlying operator, $A=-d^{2} / d x^{2}$, along with homogeneous Dirichlet boundary conditions, is well known to satisfy the resolvent estimate [31. It is also clear, since the Sobolev space $W^{1, p}(0, \pi)$ is continuously embedded in $C[0, \pi]$, that the nonlinearity fits in our framework with $\rho=1 / 2,1 \leq p \leq+\infty$.

We first discretize the problem in space by means of finite differences. To this end we fix a number $J$ of uniformly distributed nodes $x_{j}=j \Delta x, 1 \leq j \leq J$, in $(0, \pi)$, with $\Delta x=\pi /(J+1)$. The nonlinear term $\left(u^{2}\right)_{x}$ and the Laplacian operator are approximated by centered differences. Thus, we are led to consider semidiscrete problems, in the space $X_{\Delta x}=\mathbf{C}^{J}$ (endowed with the discrete $L_{p}$-norm), of the form

$$
U(t)+\partial^{-\alpha} A_{\Delta x} U(t)=U_{0}-\partial^{-1} B_{\Delta x} U(t)^{2}, \quad t \geq 0,
$$

where $A_{\Delta x}$ and $B_{\Delta x}$ are the $J \times J$ matrices

$$
A_{\Delta x}=-\Delta x^{-2} \text { tridiag }(1,-2,1), \quad B_{\Delta x}=(2 \Delta x)^{-1} \operatorname{tridiag}(1,0,-1),
$$

and, for $U \in \mathbf{C}^{J}, U^{2}$ stands for the entrywise square of $U$. As an initial condition we take $U_{0}=\left\{u_{0}\left(x_{j}\right)\right\}_{j=1}^{J}$.
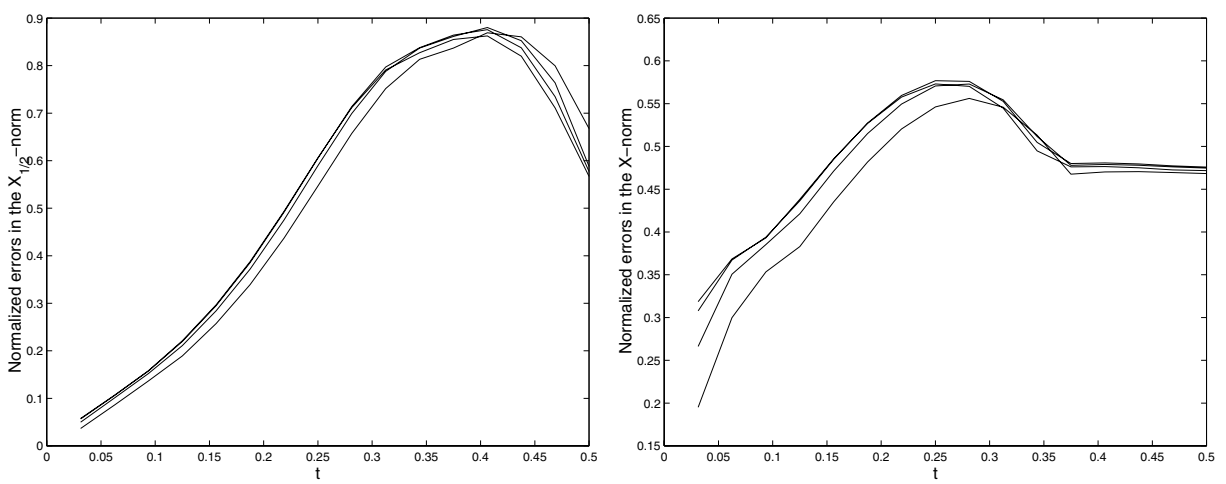

Figure 2. Left: Errors in the $X_{1 / 2}$ norm times $t^{3 / 4} / h^{2}$ vs. $t$. Right: Errors in the $X$-norm divided by $h^{2}$ vs. $t(\alpha=1.5)$. 
In order to properly focus on the time discretization, avoiding the effect of the spatial error, in the experiments we just consider the discretization in time of (9.2), keeping in mind the heuristical principle (see, e.g., Section 5 in [3]) that, for large $J$, the behavior of $\left\|U_{0}\right\|_{\rho}$ should be close to the one of $\left\|u_{0}\right\|_{\rho}$. It is known [1, 30] that $A_{\Delta x}$ satifies the basic resolvent estimate (2.2), for all $\pi / 2<\theta<\pi$.

In the second experiment we take $u_{0}(x)=\sin (x), 0 \leq x \leq \pi, J=100, \alpha=1.5$ and $p=\infty$. For $T=0.5, N=2^{k}, 4 \leq k \leq 8$, and $N_{e}=2^{10}$, we discretize (9.2) in time. Since $u_{0}$ belongs to the domain of all powers of $A$, we can apply Theorem 4.2 to (9.1) with $\rho=1 / 2$, and the same for (9.2). In Figure 2 we plot, for the different values of $h$, the errors in the $X_{1 / 2}$-norm, multiplied by $t^{\alpha \rho} h^{-2}$, and the errors in the $X$-norm, normalized by dividing them by $h^{2}$. These plots reflect a uniformly bounded error constant, as predicted by Theorem 4.2 .

9.3. Experiment 3. Again in the context of (9.2) we take $p=2, \rho=0.5, J=2000$ and

$$
u_{0}(x)=\phi(x):=c \sum_{j=10}^{J} \frac{(-1)^{j}}{j^{2.6}} \cdot \sin (j x),
$$

where $c>0$ is selected so as to have $\left\|u_{0}\right\|_{\infty}=1$. This function belongs to the domains of all powers of $A$, but $\left\|u_{0}\right\|_{2 / \alpha}$ becomes very large when $\alpha \rightarrow 1$. Since the discretization $U_{0}$ of $\phi$ is expressed in terms of eigenvectors of $A_{\Delta x}$ and the eigenvalues of $A_{\Delta x}$ are known, we are in the position to accurately estimate $\left\|U_{0}\right\|_{2 / \alpha}$, which also becomes very large when $\alpha \rightarrow 1$. On the other hand, $\left\|F_{0}\right\|_{1 / \alpha}$ is much more moderate (see the second plot in Figure 3).

The behavior of the errors is likely to be critical for small times, when the smoothing effects do not take place. Therefore, in this experiment we choose $T=$ 0.001. Now, we discretize (9.2) in time by (6.2), with $N=4$ and $N_{e}=40$. Then, for each $1 \leq \alpha<2$, we calculate the normalized error constants

$$
C(\alpha, \nu)=\max _{1 \leq n \leq 4} h^{-2} t^{\alpha \nu}\left\|U\left(t_{n}\right)-U_{n}\right\|_{\nu}, \quad \nu=0,0.5 \text {. }
$$

Theorem 6.2 predicts that $C(\alpha, \nu), \nu=0,0.5$, are bounded as long as $\left\|U_{0}\right\|_{2 / \alpha}$ and $\left\|F_{0}\right\|_{1 / \alpha}$ are bounded. Actually, in view of the second plot in Figure 3, these quantities should be bounded in terms of $\left\|U_{0}\right\|_{2 / \alpha}$. In the present experiment we represent the ratio $C(\alpha, 0.5) /\left\|U_{0}\right\|_{2 / \alpha}$ against $\alpha$ (see the left-hand plot in Figure 3 ),
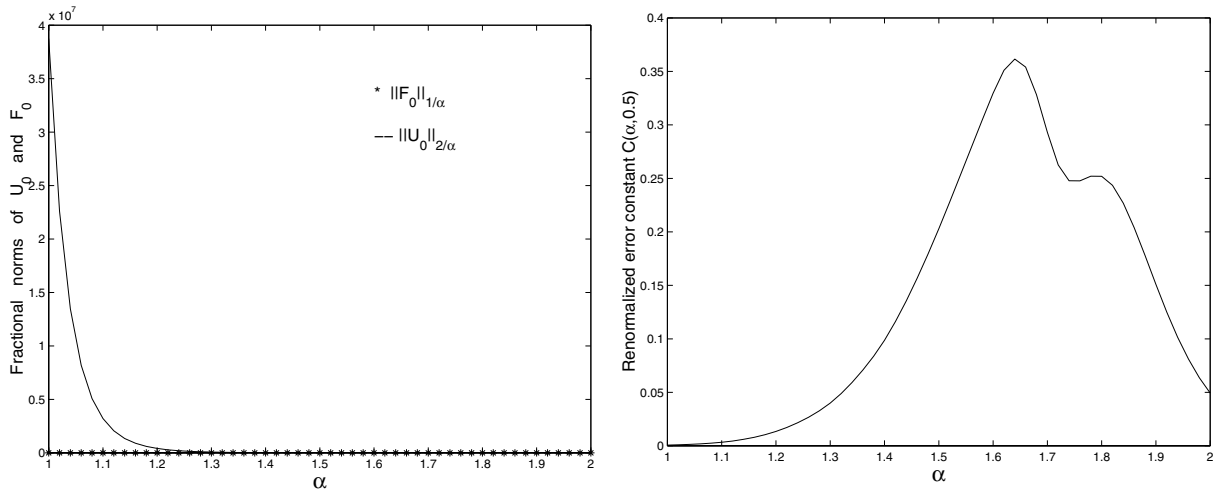

Figure 3. Left: $\left\|U_{0}\right\|_{2 / \alpha}$ and $\left\|F_{0}\right\|_{1 / \alpha}$ vs. $\alpha$. Right: Scaled error $C(\alpha, 0.5)$ divided by $\left\|U_{0}\right\|_{2 / \alpha}$ vs. $\alpha$. 

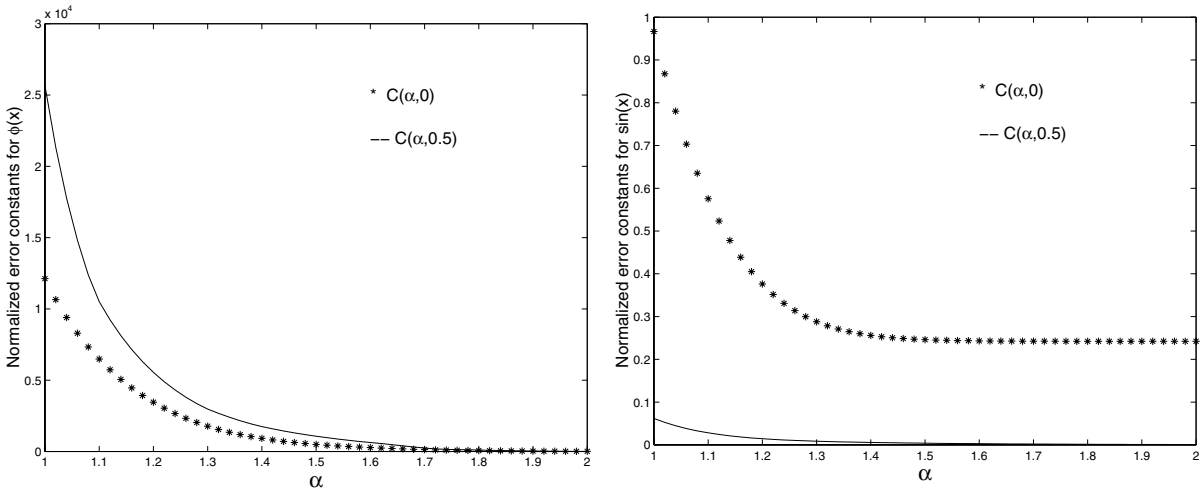

Figure 4. $C(\alpha, 0)$ and $C(\alpha, 0.5)$ vs. $\alpha$. Left: for initial data $\phi(x)$.

Right: for initial data $\sin (x)$.

which turns out to be very moderate, in spite of the fact that $C(\alpha, \nu)$ are larger than $10^{4}$ for $\alpha$ close to 1 (see the left-hand plot in Figure 4). Finally, we repeat the experiment for the initial data $u_{0}(x)=\sin x$, which belongs to the domain of all powers of $A$ with moderate norms. Now, the right-hand plot in Figure 4 shows good behavior of the error constants $C(\alpha, \nu)$ for all $\alpha$.

\section{REFERENCES}

1. A. Ashyralyev and P. Sobolevskii, Well-Posedness of Parabolic Difference Equations. Birkhäuser, Basel, 1994. MR.1299329 (95j:65094)

2. N. Y. Bakaev, V. Thomée, and L. Wahlbin, Maximum-norm estimates for resolvents of elliptic finite element operators, Math. Comp. 72 (2002) 1597-1610. MR.1986795 (2004g:65074)

3. M. P. Calvo and C. Palencia, Avoiding the order reduction of Runge-Kutta methods for linear initial boundary value problems, Math. Comp. 71 (2001) 1529-1543. MR1933043 (2003h:65091)

4. C. Chen and T. Shi, Finite Element Methods for Integro-Differential Equations. World Scientific, Singapore, 1997.

5. C. Cheng, V. Thomée, and L. Wahlbin, Finite element approximation of a parabolic integro-differential equation with a weakly singular kernel, Math. Comp. 58 (1992) 587-602. MR 1122059 (93g:65120)

6. M. Crouzeix, On multistep approximation of semigroups in Banach spaces, J. Comput. Appl. Math. 20 (1987) 25-35. MR0920377 (88j:65135)

7. E. Cuesta and C. Palencia, A fractional trapezoidal rule for integro-differential equations of fractional order in Banach spaces, Appl. Numer. Math. 45 (2003) 139-159. MR1967571 (2004b:65210)

8. E. Cuesta and C. Palencia, A numerical method for an integro-differential equation with memory in Banach spaces: Qualitative properties, SIAM J. Numer. Anal. 41 (2003) 12321241. MR2034878 (2004m:65225)

9. Y. Fujita, Integro-differential equation which interpolates the heat equation and the wave equation, Osaka J. Math. 27 (1990) 319-327. MR.1066629 (91i:45007)

10. Y. Fujita, Integro-differential equation which interpolates the heat equation and the wave equation (II), Osaka J. Math. 27 (1990) 797-804. MR1088183(92a:45032)

11. C. González and C. Palencia, Stability of Runge-Kutta methods for abstract time-dependent parabolic problems: the Hölder case, Math. Comp. 68 (1999) 73-89. MR.1609666 (99c:65108)

12. A. Hanyga, Wave propagation in media with singular memory, Math. Comput. Model. 34 (2001) 1399-1421. MR1868410 (2002m:74028)

13. D. Henry, Geometric Theory of Semilinear Parabolic Equations, LNM 840, Springer, Berlin (1981). MR0610244 (83j:35084)

14. R. Hilfer (ed.), Applications of Fractional Calculus in Physics, World Scientific, Singapore (2000). MR 1890104 (2002j:00009) 
15. M. N. Le Roux, Semidiscretization in time for parabolic problems, Math. Comp. 33 (1979) 919-931. MR0528047 (80f:65101)

16. S. Larsson, V. Thomée, and L. B. Wahlbin, Finite-element methods for a strongly damped wave equation, IMA J. Numer. Anal. 11 (1991) 115-142. MR.1089551 (92d:65164)

17. J.C. López-Marcos, A difference scheme for a nonlinear partial integrodifferential equation, SIAM J. Numer. Anal. 27 (1990) 20-31. MR1034918 (91e:65160)

18. Ch. Lubich, Discretized fractional calculus, SIAM J. Math. Anal. 17 (1986) 704-719. MR 0838249 (87f:26006)

19. Ch. Lubich, Fractional linear multistep methods for Abel-Volterra integral equations of the second kind, Math. Comput. 45 (1985) 463-469. MR0804935 (86j:65181)

20. Ch. Lubich, On the numerical solution of Volterra equations with unbounded nonlinearity, J. Integral Equations 10, Suppl. (1985) 175-183. MR0831243 (87k:65154)

21. Ch. Lubich, Convolution quadrature and discretized operational calculus. I, Numer. Math. 52 (1988) 129-145. MR0923707 (89g:65018)

22. Ch. Lubich, Convolution quadrature revisited, BIT 44 (2004) 503-514. MR.2106013 (2005f:65175)

23. Ch. Lubich and A. Schädle, Fast convolution for nonreflecting boundary conditions, SIAM J. Sci. Comp. 24 (2002), 161-182. MR1924419(2003h:44007)

24. Ch. Lubich, I.H. Sloan, V. Thomée, Nonsmooth data error estimates for approximations of an evolution equation with a positive-type memory term, Math. Comp. 65 (1996), 1-17. MR 1322891 (96d:65207)

25. F. Mainardi and P. Paradisi, Fractional diffusive waves, J. Comput. Acoustics 9 (2001) 14171436. MR $1881799(2002 \mathrm{k}: 76121)$

26. W. McLean and V. Thomée, Numerical solution of an evolution equation with a positive-type memory term, J. Austral. Math. Soc. Ser. 35 (1993) 23-70. MR1225703 (94e:65094)

27. W. McLean, V. Thomée, and L. Wahlbin, Discretizations with variable time steps of an evolution equation with a positive-type memory term, J. Comput. Appl. Math. (1996) 49-69. MR:1391611 (97b:65073)

28. R. Metzler and J. Klafter, The random walk's guide to anomalous diffusion: a fractional dynamics approach, Physics Reports 339 (2000) 1-77. MR1809268 (2001k:82082)

29. R. Metzler and J. Klafter, Accelerating Brownian motion: a fractional dynamics approach to fast diffusion, Europhys. Lett. 51 (2000) 492-498.

30. C. Palencia, The sharp resolvent estimates for the one-dimensional discrete Laplacian (in preparation).

31. A. Pazy, Semigroups of Linear Operators and Applications to Partial Differential Equations. Springer-Verlag, New York, 1983. MR0710486 (85g:47061)

32. I. Podlubny, Fractional Differential Equations. Academic Press, San Diego, 1999. MR 1658022 (99m:26009)

33. J. Prüss, Evolutionary Integral Equations and Applications. Birkhäuser, Basel, 1993. MR:1238939 (94h:45010)

34. B. Ross, Fractional Calculus, Math. Mag. 50 (1977) 115-122. MR0447500 (56:5811)

35. J. M. Sanz-Serna, A numerical method for a partial integro-differential equation, SIAM J. Numer. Anal. 25 (1988) 319-327. MR0933727 (89d:65113)

36. T. Tang, A finite difference scheme for partial integro-differential equations with a weakly singular kernel, Appl. Numer. Math. 11 (1993) 309-319. MR.1199854 (93h:65169)

37. T. Tang, A note on collocation methods for Volterra integro-differential equations with weakly singular kernels, IMA J. Numer. Anal. 13 (1993) 93-99. MR.1199031 (93k:65111)

Departamento de Matemática Aplicada, Escuela Politécnica, Universidad de Valladolid, Francisco de Mendizábal 1, 47014, Valladolid, Spain

E-mail address: eduardo@mat.uva.es

Mathematisches Institut, Universität TüBingen, Auf Der Morgenstelle 10, D-72076 Tübingen, Germany

E-mail address: lubich@na.uni-tuebingen.de

Departamento de Matemática Aplicada, Facultad de Ciencias, Universidad de Valladolid, Prado de la Magdalena s/n, 47005, Valladolid, Spain

E-mail address: palencia@mac.cie.uva.es 